\title{
The oral health-related section of the interRAl: evaluation of test content validity by expert rating and assessment of potential reasons for inaccurate assessments based on focus group discussions with caregivers
}

Stefanie Krausch-Hofmann, MSc (stefanie.krauschhofmann@kuleuven.be) ${ }^{1}$, Johanna De Almeida Mello, PhD (johanna.mello@kuleuven.be)2 , Dominique Declerck, PhD

(dominique.declerck@kuleuven.be) ${ }^{1}$, Anja Declercq (anja.declercq@kuleuven.be) ${ }^{2}$, Jan De

Lepeleire, MD, PhD (jan.delepeleire@kuleuven.be) ${ }^{3}$, Trung Dung Tran, MSc

(trungdung.tran@kuleuven.be) ${ }^{4}$, Emmanuel Lesaffre, Dr. Sc. (emmanuel.lesaffre@kuleuven.be) ${ }^{4}$, Joke Duyck,PhD (joke.duyck@kuleuven.be) ${ }^{5}$

${ }^{1}$ KU Leuven Population Studies in Oral Health - Department of Oral Health Sciences

Kapucijnenvoer 7/a - box 7001

3000 Leuven, Belgium

${ }^{2}$ KU Leuven LUCAS - Centre for Care Research and Consultancy

Minderbroedersstraat 8 - box 5310

3000 Leuven, Belgium

${ }^{3}$ KU Leuven Academic Centre for General Practice - Department of Public Health and Primary

Care

Kapucijnenvoer 33/j - box 7001

3000 Leuven, Belgium

${ }^{4}$ KU Leuven Biostatistics and Statistical Bioinformatics Centre (L-BioStat) - Department of Public Health and Primary Care

Kapucijnenvoer 35/d - box 7001

3000 Leuven, Belgium

${ }^{5}$ Biomaterials/BIOMAT - Department of Oral Health Sciences

Kapucijnenvoer 35/d - box 7001

3000 Leuven, Belgium

Corresponding author:

Stefanie Krausch-Hofmann

Population Studies in Oral Health - Department of Oral Health Sciences

Kapucijnenvoer 7/a - box 7001

3000 Leuven, Belgium

E-mail: stefanie.krauschhofmann@kuleuven.be 


\title{
The oral health-related section of the interRAl: evaluation of test content validity by expert rating and assessment of potential reasons for inaccurate assessments based on focus group discussions with caregivers
}

\begin{abstract}
Objectives: To explore the failure of the oral health-related section of the interRAI (ohr-interRAI), this study investigated test content validity (A.) and reasons for inaccurate assessments (B.).

Background: Poor oral health negatively affects quality of life and is associated with a number of systemic diseases. The interRAI instruments, internationally used for geriatric assessment, should accurately detect oral conditions that require care. Previous research showed that the ohr-interRAI and related precursor versions do not achieve this goal.

Materials and Methods: (A.) A group of 12 experts rated completeness, relevance, clarity of wording and feasibility of the ohr-interRAI. Content validity indices were calculated per item (threshold 0.78). (B.) Focus group discussions with 23 caregivers were organized. A semi-structured question guide made sure that all topics of interest were covered. Qualitative content structuring analysis was applied after transcription.
\end{abstract}

Results: (A.) Experts agreed on the relevance of the items on chewing, pain, gingival inflammation and damaged teeth. They regarded none of the items as worded clearly and only prosthesis use and pain were considered to be assessable by untrained caregivers. All experts agreed that the ohrinterRAI was incomplete. (B.) Focus group discussions revealed that in the care environment oral health had low priority. Aspects related to the ohr-interRAI itself and aspects related to the assessment situation impeded the oral health assessment. The approach of the caregivers to complete the ohr-interRAI was inappropriate to accurately detect oral care needs.

Conclusions: Findings challenge test content validity of the ohr-interRAI and reveal reasons for inaccurate assessments.

\section{Keywords}

InterRAI; geriatric assessment; oral health; mouth diseases; focus groups; caregivers; validity; test 
content; inaccurate assessments

\section{Introduction}

The interRAI suite of instruments is used internationally for comprehensive assessment of caredependent individuals ${ }^{1,2}$. A first version of the instrument, the Resident Assessment Instrument Minimum Data Set (RAI-MDS 1.0), was developed and tested between 1987 and 1989 and implemented in 1990 in the United States. It assessed concerns, strengths and needs of caredependent nursing home residents on multiple dimensions ${ }^{3}$. In the following years, versions for other health-care settings (Home Care, Acute Care, et cetera) were released, psychometric properties of the constituting components were evaluated and the instruments were updated regularly. In 2005 the restructured interRAI suite of instruments was released by the international interRAI consortium to facilitate compatibility of the versions available for the different health-care settings ${ }^{1}$.

Based on the assessment, scaled outcome measures are calculated that assist to determine the condition and needs of care dependent individuals. Furthermore, algorithms are used to determine whether a certain Clinical Assessment Protocol (CAP) is triggered or not. These triggers indicate specific risks or resources and help to prioritize and specify individuals care needs ${ }^{1,4}$.

The present study focuses on the oral health-related section that is available in the interRAI version for Long-Term Care Facilities (LTCF) and for Home Care (HC), respectively. Care-dependent individuals often lack the cognitive and physical abilities to perform adequate oral hygiene and to access professional dental care ${ }^{5,6}$. As a result, oral health of care-dependent individuals is generally poor ${ }^{7,8}$. Compromised oral health causes pain and discomfort, which in turn is related to low oral health-related quality of life ${ }^{9,10}$. It is also associated with a number of systemic diseases ${ }^{11-13}$, deteriorated physical ${ }^{14-16}$ and cognitive performance ${ }^{17-19}$, depression ${ }^{20}$, institutionalization ${ }^{21}$, and even mortality ${ }^{22,23}$ in frail individuals. This emphasizes the need of a thorough and accurate assessment of oral health which in turn facilitates planning of daily oral care and the referral for professional treatment if needed.

To evaluate the quality of data based on the ohr-interRAI, psychometric properties need to be 
examined. Inferences are meaningful, appropriate, and useful only if the instrument is valid. Validity is composed of different aspects that each require particular validation activities: test content, response process, internal structure, relations to other variables, and consequences of testing. Accumulated results allow to indicate if the ohr-interRAl is valid ${ }^{24,25}$.

A study based on home care data from 7590 individuals in Belgium revealed a substantial proportion of about $17 \%$ missing data for the ohr-interRAI. Prevalence of missing data was higher in clients with cognitive impairment or depression ${ }^{26}$. Missing data challenge test content validity of the ohr-interRAI as they might be caused by unobtainable information, misinterpretation, or low relevance of oral health on part of the assessors ${ }^{25}$.

Other studies on validity were based on the oral health-related section of the Resident Assessment Instrument - Minimum Data Set 2.0 (ohr-RAI-MDS 2.0) which is the predecessor of the ohr-interRAI. Folse (2001) compared ohr-RAI-MDS 2.0 data of about 3.6 million subjects to data retrieved from dental examination forms in the United States. Pain in the mouth was recorded in $0.8 \%$ of the ohrRAI-MDS 2.0 data, although $56.1 \%$ of the dental examinations revealed pain. The same trend was seen for other oral health-related variables ${ }^{27}$. Based on ohr-RAI-MDS 2.0 measurements from about 2700 institutionalized individuals in Canada, Hoben et al. (2016) as well found a substantial underdetection of oro-dental problems when compared to prevalence data that were based on oral examinations. Well-known associations between predictors and oro-dental problems were not found in the RAI-MDS 2.0 data ${ }^{28}$. In a study with about 200 institutionalized subjects from Sweden, treatment need was not identified correctly by the ohr-RAI-MDS 2.0 when compared to professional oral examinations for $50 \%$ of the participants ${ }^{29}$. Accordingly, of the 236 Directors of Nursing that participated in the study of Ettinger et al. (2000) only 9\% stated that the ohr-RAI-MDS 2.0 was often useful in identifying dental problems ${ }^{30}$. Although Arvidson-Bufano et al. (1996) showed that a short training session can improve the ability of caregivers to assess oral health ${ }^{31}$, study results above indicate that validity based on correlation with external variables is poor for the ohr-RAI-MDS 2.0. Guay (2005) had challenged content validity ${ }^{32}$ and Hawes et al. (1995) reported a low average interrater reliability of 0.46 for the oral health-related items ${ }^{33}$.

The ohr-interRAI is a shorter, slightly modified version of the ohr-RAI-MDS 2.0 [see additional file 1 
for an overview of the different versions and where they are used] and the findings mentioned above raise the question whether the instrument is adequate to detect care-requiring oral health-related conditions.

The overall objective of our research project is the development and validation of an optimized ohrinterRAI. As a preparatory step, limitations of the current instrument need to be investigated more comprehensively. The current study consisted of two equivalent parts that each included a distinct group of participants. In the first part, test content validity was evaluated based on expert rating. It was determined whether the current items were considered complete, relevant, clearly worded and feasible to detect oral health-related treatment need. The second part of the study focused on potential reasons for inaccurate oral health-related assessments with the ohr-interRAI. Based on focus group discussions with caregivers who were acquainted with the use of the ohr-interRAl it was evaluated whether their attitude and approach of data collection was suitable to detect oral healthrelated treatment need.

\section{Methods}

\section{Ohr-interRAl}

The interRAI Long-Term Care Facility (LTCF) instrument dichotomously ( $0=$ no, $1=y e s)$ registers oral health-related problems occurring in the three days prior to the assessment. The following items are included:

- Wears a denture/removable prosthesis (prosthesis use)

- Has broken, fragmented, loose, or otherwise non-intact natural teeth (damaged teeth)

- Reports having dry mouth (dry mouth)

- Reports difficulty with chewing (chewing difficulty)

- Reports mouth or facial pain/discomfort (pain)

- Presents with gum (soft tissue) inflammation or bleeding adjacent to natural teeth or tooth fragments (gingival inflammation)

Pain and gingival inflammation are not included in the Home Care $(\mathrm{HC})$ version. The interRAI utilization guidelines give a short definition of each item. The instructions advise the assessors to collect the information by interview, observation during meals, or inspection of the mouth. 


\section{InterRAI in Belgium}

In 2016 the Belgian government decided to incrementally mandate the use of the instruments that in Belgium are named BeIRAI ${ }^{34}$. Currently they are in the state of implementation and applied in multiple pilot projects in different health care settings ${ }^{35}$. Depending on the individual care facility, the instruments are either completed multidisciplinary or by a single caregiver ${ }^{36}$. Assessors receive three days of training on how to complete the interRAl instrument ${ }^{37}$. Based on reports from instructors and training participants, the ohr-interRAl does not receive special attention during training sessions.

\section{A. Expert rating to evaluate test content validity}

The ideal ohr-interRAI should identify clients who need to be referred to a dentist or who need help with their daily oral hygiene ${ }^{8}$. By investigating test content validity this part of the study examines, to what extent items are relevant, clearly worded, feasible and complete to be considered adequate for this goal. Expert rating is a procedure that is widely accepted and used to analyze test content validity ${ }^{25,38-40}$.

In October 2015 an expert meeting was organized in Flanders, Belgium. The 12 experts that were invited agreed to take part. Experts were chosen based on their academic and clinical professional background relevant for oral health of frail individuals. The group comprised three university professors in gerodontology, two experienced dentists working in long-term care facilities, one periodontologist, three geriatricians and one college lecturer in geriatric nursing care. Two experts one university professor and one experienced dentist - were specialized in prosthetic dentistry.

Using the method outlined by Lynn (1986), relevance of each item of the ohr-interRAl was quantified in a standardized way ${ }^{38}$. A questionnaire was provided to the experts who rated each item on a 4point Likert scale $(1=$ not relevant, 2 = somewhat relevant, $3=$ quite relevant, $4=$ highly relevant). The original method was extended to evaluate further limitations of the items, even if basically the experts considered them relevant. Clarity of wording was assessed per item and the associated utilization guidelines $(1=$ not clearly formulated, 2 = somewhat clearly formulated, 3 = quite clearly formulated, 4 = very clearly formulated). Additionally, expert opinion on feasibility was evaluated, rating whether the items can be completed by caregivers who were not specifically trained for the 
ohr-interRAI ( 1 = not feasible, 2 = somewhat feasible, 3 = quite feasible, 4 = very feasible). For each item, relevance, clarity of wording, and feasibility was quantified using the content validity index (CVII) that defines the proportion of favorable rankings (3 or 4 ) over the total rankings on item-level ${ }^{38}$. As recommended by Polit et al. (2007), 0.78 was used as a threshold ${ }^{40}$.

Experts also dichotomously (yes/no) assessed the completeness of the list of items.

\section{B. Focus group discussions with caregivers to evaluate potential reasons for inaccurate assessments}

Accurate assessments are threatened when caregivers are not aware of the relevance of oral health and do not seriously attempt to complete the items correctly. Problems caused by the ohr-interRAI instrument itself or challenges related to the assessment situation might also impede accurate assessments. A qualitative approach was chosen to make sure that the whole range of opinions and experiences could be explored. The social interaction of the focus group discussions encourages participants to think about the topic deeply and to clarify individual and shared perspectives ${ }^{41,42}$.

\section{Development of the question guide}

A semi-structured question guide was designed to direct the discussions in a way that all topics of interest would be covered. The guide was designed by one researcher based on the recommendations of Krueger \& Casey (2000) ${ }^{41}$. It was revised during two meetings of the research group. It was agreed that the first focus group would also be regarded as a pilot test for the question guide.

To initiate the discussion, participants were asked to reflect about oral health-related activities provided by the employing care organizations. They were also invited to share their opinion on the relevance of oral health compared to other care needs of the clients. Furthermore, the question guide covered the following points of interest:

- Attitudes and knowledge related to oral health and hygiene.

- Attitudes and problems related to the ohr-interRAI.

- Approach to complete the ohr-interRAI.

- Reasons to not complete the ohr-interRAI (missingness).

- Perceived competency to complete the ohr-interRAI.

- Suggestions for an optimized version of the ohr-interRAI. 
The moderator was also open for additional topics that were brought up by the participants.

To gain insight in their personal oral health-related behavior, participants were asked to fill in a short questionnaire that included the history of dental extractions (excluding wisdom teeth), dental emergency consults during the previous year, frequency of annual dental check-up's, and frequency of daily tooth brushing.

\section{Recruitment of participants}

Recruitment of focus group participants was based on a complete list of 102 care projects/organizations in Flanders, Belgium, that used or had used the interRAI-LTCF or -HC. The 76 responsible management executives were contacted by e-mail and by phone and the objectives of the study were explained. They were invited to send one caregiver who actually completed or had completed the ohr-interRAI on a daily basis. No further inclusion- or exclusion criteria were applied. Six contacts could not be reached and participation was refused for 65 projects (Table 1).

Non-participation was predominantly motivated by other priorities and low relevance of the topic. Cessation of interRAI related care projects was also mentioned as explanation for refusal. From the initial 31 participants, 8 dropped out due to illness or other reasons on the day of the discussion.

\section{Practical organization}

In March 2016, four focus group discussions were organized at different locations in Flanders. The discussions took place in the meeting room of a care facility and took approximately two hours. Participants completed the questionnaire on their personal oral health-related behavior before starting the discussions. The background and goals of the research project and of the involved researchers were introduced to the participants. A trained researcher $(\mathrm{SKH})$ moderated the groups, guided by the semi-structured question guide. The discussions were conducted in the Flemish Dutch language. A co-moderator (JD) took field notes and assisted with the practical organization. Only participants and researchers were present during the focus groups. All discussions were audio recorded.

\section{Analysis}

Discussions were transcribed verbatim. For readability, dialect was translated to standard language and filling words were removed. Para- and non-verbal communication was excluded from the analysis. Coding was done by one researcher (SKH) and validated by another member of the group 
(JDAM). Code differences were resolved by discussion between the two researchers. The software program NVivo 11 was used to manage data and to support the coding process.

Qualitative analysis was based on structuring content analysis - also called theme analysis - that combines deductive and inductive coding activities. Main categories were pre-determined by the topics of interest that were also translated to the question guide. First, the material was coded based on these categories. In a second step, further differentiation of the codes was based on the material itself 43 .

\section{Results}

\section{A. Expert rating to evaluate test content validity}

Table 2 shows the content validity indices (CVI) that quantify the proportion of favorable expert rankings over total rankings for relevance, clarity of wording, and feasibility for each item of the ohrinterRAI. Experts unanimously agreed on the relevance of chewing difficulty, pain and gingival inflammation. Based on the 0.78 threshold, damaged teeth was also considered relevant. This was not the case for prosthesis use and dry mouth. None of the items - and the corresponding utilization guidelines - was considered clearly worded by the experts. Pain was assessed nearly satisfactorily. Out of the six items, only prosthesis use and pain were considered feasible to be completed by care givers not particularly trained for the ohr-interRAl. All experts agreed that the list of items was incomplete.

\section{B. Focus group discussions with caregivers to evaluate potential reasons for inaccurate assessments}

The average age of the 23 participants (20 female) was 39.8 years (range $25-53$ years). While 15 participants had worked with the interRAI-HC, 5 were acquainted with the interRAI-LTCF, and 3 participants had worked with both versions. The participants had varying professional backgrounds, for example, nurses, quality-of-care coordinators, occupational therapists and social assistants. With regard to their personal oral health behavior, all participants reported annual dental check-ups and daily tooth brushing. More than half of the participants had no history of tooth extractions (except wisdom teeth) and only three had a dental emergency consult in the previous year [additional file 2]. For the four focus group discussions the same question guide was applied. Table 3 presents discussion contributions of caregivers after structuring content analysis. Selected contributions 
relevant to discuss potential reasons for inaccurate assessments of the ohr-interRAl are summarized below.

Attitudes and knowledge concerning oral health and hygiene: In general and on a personal level, oral health and hygiene were considered to be important. Participants were aware of the impact on general health and on social relationships. However, in the daily care-environment and for clients themselves, priority of oral health was low compared to other problems.

Citation: When time is short, oral care is the first task that is omitted.

This was attributed to low visibility of the results of oral care and aversion towards the oral area of clients.

Complaints and criticism concerning the ohr-interRAl: Participants considered oral health and hygiene uncommon and sensitive topics of discussion. They mentioned that the assessment might evoke feelings of embarrassment and fear.

Citation: Oral health is not a normal topic of conversation, especially not during a first meeting.

It was further criticized that the ohr-interRAI fails to register oral health-related information that was considered important by the participants. For example, oral hygiene, soft tissue lesions, and functionality and fitting of dental prostheses.

Citation: Are they able to brush their teeth and are teeth clean after brushing? Are clients aware that they have to take out their dental prosthesis for cleaning? Do they accept if caregivers do it for them?

Participants also missed an outcome measure based on the ohr-interRAl that would allow interpretation of registrations and emphasize the importance of the oral health assessment. It was further mentioned that the item on gingival inflammation should be removed, as detection by caregivers was not considered feasible.

Situational factors that challenge the assessment of oral health: Participants mentioned that a short contact time with clients impaired the establishment of a trustful relationship that was needed to assess oral health. Client characteristics and needs also played a role, for example, poor communication skills. Completion of the ohr-interRAl was considered inappropriate during a first assessment or during a crisis assessment, when clients performed oral hygiene independently and 
when oral health was not relevant for clients.

Citation: It is the same with shoes. When I ask, is it important for you that your shoes are clean and you say no, I will not inquire, when for the last time you have cleaned them. I will just think, OK, never mind.

The background of the assessors and the health-care setting played a role as well. Completion of the ohr-interRAI was considered inappropriate when the organization the caregiver was employed by did not provide daily oral care and when clients with oral care needs were not referred to professional treatment. Some professions were considered to be less common to work with the mouth. Based on their education, competencies and anticipated client expectations, assessment of oral health seemed inappropriate.

Citation: I am a social assistant - I cannot ask them if there is a problem with their teeth.

Approach to complete the ohr-interRAl: In general, the ohr-interRAl was completed hastily and superficially. Clients were observed during conversation, mealtime or care-giving. Completion was also based on conversation. Participants preferred to talk to primary caregivers and oral healthrelated questions were softened by harmless chatting. Inspection of the mouth was regarded disrespectful, inappropriate, and devaluating and the mouth was considered an intimate area of the body.

Citation: Ask somebody to open the mouth - that is a question you never ask another person. One does not do that, you have to respect other people's dignity.

It was also mentioned that item wording implied active reporting of oral-health related problems by clients.

Missing values due to non-completion of the ohr-interRAl: In general, participants attempted to complete the whole interRAI as this was requested by the employing care-organizations. However, non-completion of the ohr-interRAI was considered inevitable when items were not applicable. For example, the item on chewing problems was left open when nutrition was exclusively provided via a feeding-tube or when clients were comatose. Participants also mentioned that items were not completed when the assessment was considered unfeasible, for example, detection of damaged teeth.

Perceived competency to complete the ohr-interRAl: In general, items were considered clear and 
participants felt no need for training. However, it was mentioned that the assessment of damaged teeth, dry mouth, and gingival inflammation was challenging.

\section{Discussion}

The ideal ohr-interRAI should detect clients with oral health-related treatment needs. Previous research indicated that the current instrument and related precursor versions do not achieve this goal ${ }^{27-30}$. The present study investigated the underlying problems of this failure by examining test content validity and potential reasons for inaccurate assessments with the ohr-interRAI.

\section{A. Expert rating to evaluate test content validity}

An oral assessment instrument that is used by caregivers should identify clients who need help with their daily oral hygiene or who need to be referred to a dentist for further diagnosis and treatment ${ }^{8}$. Experts agreed unanimously that items of the ohr-interRAl were not complete to achieve this goal. For example, registration of oral hygiene is essential as plaque accumulation is the basis for dental decline and correlated to respiratory disease ${ }^{11,44-46}$. It also needs to be clarified why gingival infection and pain are registered in the interRAI-LTCF, but not in the -HC version.

Rating of individual items showed that experts considered damaged teeth, chewing difficulty, pain, and gingival inflammation relevant for the ohr-interRAI. The American Dental Association (ADA) had recommended these items to be included in the ohr-RAI-MDS 2.0 as well ${ }^{32}$. Their relevance was also confirmed by numerous publications that found associations with either general health or oral health-related quality of life in older individuals $9,12,13,19,47-60$. Registration of prosthesis use was not considered relevant by the experts. Non-adequate functionality and fitting of prosthesis might instead be more important for the decision whether clients need to be referred to a dentist ${ }^{61-63}$. Recommendations of the ADA also suggested to register problems related to the prosthesis ${ }^{32}$. Dry mouth was not considered relevant by the experts, although literature showed that both hyposalivation (insufficient production of saliva) and xerostomia (perception of dry mouth) affected oral health and quality of life ${ }^{64-66}$. However, dry mouth does not have to be a problem if oral hygiene is adequate and if oral perception of the client is not negatively affected. ADA recommendations also did not suggest dry mouth to be included in the ohr-RAI-MDS 2.0. ${ }^{32}$.

Experts challenged clarity of wording for all items of the ohr-interRAI. This might be attributed to the 
unspecified method of data collection. While a number of items are worded as 'Client reports' implying a verbal assessment - guidelines invite assessors to complete the section based on observation, interview or inspection. Suitability of data for research was doubted due to this vague description of the assessment procedure ${ }^{67,68}$. Dry mouth received the lowest rating for clarity of wording. This might be caused by the ambiguity whether hyposalivation or xerostomia should be detected. Literature showed that the relationship between both phenomena is complex ${ }^{69}$.

Registration of prosthesis use was considered feasible by the experts, probably as the presence of a removable prosthesis can be assessed easily. Pain was rated feasible as well and chewing difficulty was rated nearly feasible to be assessed by untrained caregivers. Clients can be asked about the presence of these symptoms, however, clear instructions are lacking for the assessment of clients who are unable to communicate. Feasibility of the current ohr-interRAI to detect damaged teeth, dry mouth, and gingival inflammation was doubted by the experts. These findings on feasibility are in line with the results of Chalmers et al. (2005) who had invited caregivers to share their experiences concerning the completion of the Oral Health Assessment Tool - a validated oral screening instrument ${ }^{70}$. The ADA had already questioned test content of the ohr-RAI-MDS 2.0 and suggested a revised version of the instrument ${ }^{32}$. However, it was unclear how test content validity was assessed and how the modified version was developed. In the present study a structured method was applied to evaluate test content validity. The assessment of item relevance was further complemented by the evaluation of clarity of wording, feasibility and completeness to allow for a more detailed view.

\section{B. Focus group discussions with caregivers to evaluate potential reasons for inaccurate assessments}

The second part of the study explored whether attitudes and approach of caregivers who complete the instrument were suitable to detect oral health-related treatment need. Personal oral healthrelated behavior of participants showed that the majority had only their wisdom teeth extracted, had annual dental check-ups and practiced dental hygiene on a regular basis. Participants were also aware of the relevance of oral health on a general and on a personal level. These findings support a thorough completion of the ohr-interRAI. It has been reported that health promotion and provision 
of care was influenced by caregivers' own behaviors, attitudes, priority, and awareness towards health ${ }^{71,72}$. In the routine of the care environment, however, oral health had low priority compared to other problems. This was confirmed by other studies that evaluated attitudes of caregivers towards oral health of clients ${ }^{71,73,74}$. Low relevance of oral health on part of the clients might be a major cause of this divergence of priority. This is also reflected by the perception of inappropriateness when the ohr-interRAI had to be completed for clients for whom oral health was not important. It was reported in the literature as well that respect for the self-determination of clients was an impediment for caregivers to provide daily oral care ${ }^{71}$. Oral health was further considered a sensitive and uncommon topic which is an additional threat for a thorough completion of the ohr-interRAI.

Participants also expressed their complaints with regard to the instrument itself, for example, lack of an item that registers oral hygiene. Findings related to missingness caused by non-completion revealed further deficits such as the absence of a non-applicable response option. But if caregivers perceive the ohr-interRAI as not valuable to detect oral health-related care-needs, their motivation to make effort to complete the instrument could be compromised. Psychological research confirmed that low task significance was negatively associated with work motivation and performance ${ }^{75}$. Participants further criticized that it was not feasible for caregivers to detect gingival inflammation and damaged teeth. This position also challenges a thorough completion of the ohr-interRAl as it was shown that poor self-efficacy reduces the effort individuals exert on a given task ${ }^{76}$. The study further revealed a number of situational factors that challenged the oral health assessment. This indicates a lack of training that conveys the skills how oral health has to be assessed accurately and how challenges can be dealt with. Instead, the general approach to complete the ohr-interRAI was hasty and superficially. A further threat to an accurate oral health assessment is, that the approach to complete the instrument did not include an oral inspection. Previous research revealed an extensive discrepancy between normative and self-perceived oral health-related needs in older individuals ${ }^{6}$ and the value of a superficial observation during meals or care-giving is highly questionable. However, participants considered an oral inspection of the client inappropriate and the mouth was perceived an intimate area. It was reported previously that for caregivers the mouth was an intimate region of the body ${ }^{77}$. 
In summary, findings of the second part of the study indicate potential reasons for inaccurate assessments. The adequate detection of oral health-related treatment needs is impeded by shortcomings of the instrument and a lack of clear guidelines and training. It has been reported in the literature that even a short training enhanced the ability of caregivers to assess the oral health of clients ${ }^{31}$. The training should not be limited to the acquisition of skills, but also raise awareness of the interrelation between oral health, other health aspects, and quality of life.

It is a strength of the present study that two distinct aspects of the ohr-interRAl were investigated. Findings of the two parts complement each other and provide a detailed view of the limitations related to the current instrument and how this is used. These results are valuable for the development of an optimized ohr-interRAI, which is the follow-up project of this research. As an integrated part of the interRAI assessment, the optimized ohr-interRAI should briefly register relevant oral health aspects. It should include questions to evaluate subjective oral health complaints, but also mandate an oral examination.

It is a limitation of the present study that among the experts, groups who complete the items were under-represented, for example nursing scientists. Their expertise would be valuable to more validly assess feasibility of the items. It further needs to be mentioned that the second part of the study did not include the perspective of other parties, for example, clients or management executives. However, caregivers are the most relevant group, as they actually complete the ohr-interRAI. In an early phase of the current study it was planned to conduct focus group discussions with management executives, but these were not aware of the details related to the completion of the ohr-interRAI. The authors also consider to investigate the perspective of the clients in a later stage of the project when the optimized ohr-interRAI will be validated. Another limitation that needs to be discussed is that only a small proportion of the contacted care-organizations sent a participant to the focus group discussions. This reflects and confirms the finding that oral health has only low priority in the care environment. Recruitment of participants required approval of their management executives who had to consider oral health a topic relevant enough worth the expenditure of time and effort. Data saturation is the gold standard for qualitative research ${ }^{76}$ and we can only speculate whether further respondents would have added additional insights. Future research - preferably on a larger and 
international scale - could clarify if results of the current study are exhaustive and generalizable.

\section{Conclusions}

The presented study contributes to the understanding why the existing ohr-interRAl and related precursor versions fail to adequately assess oral health-related treatment need ${ }^{26-30}$. Findings challenge test content validity and reveal potential reasons for inaccurate assessments. In a followup project the present results will be integrated to develop an optimized and validated ohr-interRAI and an associated training tool.

\section{Declarations}

\section{Ethics approval and consent to participate}

In July 2015 the protocol of the present study was sent to the Social and Societal Ethics Committee of the KU Leuven. We received the response that - as participants were not subjected to tests or experiments - approval of the committee was not required. The study adhered to standard ethical guidelines as detailed study information for participants, voluntariness of participation and guaranteed privacy. Participants were aware and consented that results of the study will be published.

\section{Competing interests}

The authors declare that they have no competing interests.

\section{Funding}

This study was part of a research project financed by an internal fund of the KU Leuven (C24/15/034). Study design, analysis, interpretation and presentation of results was not influenced by the funding source.

\section{Acknowledgements}

The authors would like to thank all participants for sharing their experience and knowledge.

\section{References}

1. InterRAI. InterRAI Instruments. http://www.interrai.org/instruments/. Accessed March 3, 2019.

2. Vanneste D, Declercq A. The Development of BeIRAI, a Web Application for Sharing Assessment Data on Frail Older People in Home Care, Nursing Homes, and Hospitals.; 2014. doi:10.4018/978-1-4666-6138-7

3. Morris JN, Hawes C, Fries BE, et al. Designing the National Resident Assessment 
Instrument for Nursing Homes. Gerontologist. 1990;30(3):293-307. doi:10.1093/geront/30.3.293

4. Gray LC, Bernabei R, Berg K, et al. Standardizing assessment of elderly people in acute care: the interRAI Acute Care instrument. J Am Geriatr Soc. 2008;56(3):536-541. doi:10.1111/j.1532-5415.2007.01590.x

5. Jablonski RA, Kolanowski A, Therrien B, Mahoney EK, Kassab C, Leslie DL. Reducing careresistant behaviors during oral hygiene in persons with dementia. BMC Oral Health. 2011;11(1):30. doi:10.1186/1472-6831-11-30

6. Niesten D, van Mourik K, van der Sanden W. The impact of frailty on oral care behavior of older people: a qualitative study. BMC Oral Health. 2013;13(1):61. doi:10.1186/1472-683113-61

7. Visschere L, Janssens B, Reu G, Duyck J, Vanobbergen J. An oral health survey of vulnerable older people in Belgium. Clin Oral Investig. 2016;20:1903-1912. doi:10.1007/s00784-015-1652-8

8. Chalmers JM, Pearson A. A systematic review of oral health assessment by nurses and carers for residents with dementia in residential care facilities. Spec Care Dentist. 25(5):227233. doi:10.1111/j.1754-4505.2005.tb01654.x

9. Porter J, Antiopi N, Read A, Murdoch M, Ola D, Tsakos G. The impact of oral health on the quality of life of nursing home residents. Health Qual Life Outcomes. 2015. http://dx.doi.org/10.1186/s12955-015-0300-y.

10. Locker D, Matear D, Stephens M, Lawrence H, Payne B. Comparison of the GOHAl and OHIP-14 as measures of the oral health-related quality of life of the elderly. Community Dent Oral Epidemiol. 2001;29(5):373-381. http://www.ncbi.nlm.nih.gov/pubmed/11553110. Accessed February 12, 2015.

11. Vilela MCN, Ferreira GZ, Santos PSDS, Rezende NPM De. Oral care and nosocomial pneumonia: a systematic review. Einstein (Sao Paulo). 2015;13(2):290-296. doi:10.1590/S1679-45082015RW2980

12. Borgnakke WS, Ylöstalo P V, Taylor GW, Genco RJ. Effect of periodontal disease on diabetes: systematic review of epidemiologic observational evidence. J Periodontol. 2013;84(4 Suppl):S135-52. doi:10.1902/jop.2013.1340013

13. Teeuw WJ, Slot DE, Susanto $\mathrm{H}$, et al. Treatment of periodontitis improves the atherosclerotic profile: a systematic review and meta-analysis. J Clin Periodontol. 2014;41(1):70-79. doi:10.1111/jcpe.12171

14. Moriya S, Notani K, Miura H, Inoue N. Relationship between masticatory ability and physical performance in community-dwelling edentulous older adults wearing complete dentures. Gerodontology. 2014;31(4):251-259. doi:10.1111/ger.12029

15. Moriya S, Tei K, Yamazaki Y, et al. Relationships between perceived chewing ability and muscle strength of the body among the elderly. J Oral Rehabil. 2011;38(9):674-679. doi:10.1111/j.1365-2842.2011.02207.x

16. Moriya S, Tei K, Murata A, Sumi Y, Inoue N, Miura H. Influence of dental treatment on 
physical performance in community-dwelling elderly persons. Gerodontology. September 2012:no-no. doi:10.1111/j.1741-2358.2011.00563.x

17. Teixeira FB, Pereira Fernandes LDM, Noronha PAT, et al. Masticatory deficiency as a risk factor for cognitive dysfunction. Int J Med Sci. 2014;11(2):209-214. doi:10.7150/ijms.6801

18. Weijenberg RAF, Scherder EJA, Lobbezoo F. Mastication for the mind--the relationship between mastication and cognition in ageing and dementia. Neurosci Biobehav Rev. 2011;35(3):483-497. doi:10.1016/j.neubiorev.2010.06.002

19. Kaye EK, Valencia A, Baba N, Spiro A, Dietrich T, Garcia RI. Tooth loss and periodontal disease predict poor cognitive function in older men. J Am Geriatr Soc. 2010;58(4):713-718. doi:10.1111/j.1532-5415.2010.02788.x

20. Laudisio A, Milaneschi Y, Bandinelli S, Gemma A, Ferrucci L, Incalzi RA. Chewing problems are associated with depression in the elderly: Results from the InCHIANTI study. Int $J$ Geriatr Psychiatry. 2014;29(3):236-244. doi:10.1002/gps.3995

21. Moriya S, Tei K, Murata a, Muramatsu M, Inoue N, Miura H. Perceived chewing ability and need for long-term care in the elderly: a 5-year follow-up study. J Oral Rehabil. 2012;39(8):568-575. doi:10.1111/j.1365-2842.2012.02302.x

22. Osterberg T, Carlsson GE, Sundh V, Mellström D. Number of teeth--a predictor of mortality in 70-year-old subjects. Community Dent Oral Epidemiol. 2008;36(3):258-268. doi:10.1111/j.1600-0528.2007.00413.x

23. Hämäläinen $\mathrm{P}$, Meurman $\mathrm{JH}$, Kauppinen $\mathrm{M}$, Keskinen M. Oral infections as predictors of mortality. Gerodontology. 2005;22(3):151-157.

24. American Educational Research Association, American Psychological Association, National Council on Measurement in Education. Standards for Educational and Psychological Testing. 6th ed. Washington, DC; 2014.

25. Wellens NIH, Milisen K, Flamaing J, Moons P. Methods to assess the validity of the interRAI Acute Care: a framework to guide clinimetric testing. J Eval Clin Pract. 2012;18(2):296-306. doi:10.1111/j.1365-2753.2010.01571.x

26. Krausch-Hofmann S, Bogaerts K, Hofmann M, et al. Missing Oral Health-Related Data in the interRAI-HC - Associations with Selected Variables of General Health and the Effect of Multiple Imputation on the Relationship between Oral and General Health. PLoS One. 2015;10(12):e0146065. doi:10.1371/journal.pone.0146065

27. Folse GJ. National MDS and dental deficiency data reported by the US Health Care Financing Administration (HCFA). Spec Care Dentist. 2001;21(1):37-38.

28. Hoben M, Poss JW, Norton PG, Estabrooks CA. Oral/dental items in the resident assessment instrument - minimum Data Set 2.0 lack validity: results of a retrospective, longitudinal validation study. Popul Health Metr. 2016;14(1):36. doi:10.1186/s12963-0160108-y

29. Nordenram G, Ljunggren G. Oral status, cognitive and functional capacity versus oral treatment need in nursing home residents: a comparison between assessments by dental and ward staff. Oral Dis. 2002;8(6):296-302. 
30. Ettinger RL, O'Toole C, Warren J, Levy S, Hand JS. Nursing directors' perceptions of the dental components of the Minimum Data Set (MDS) in nursing homes. SpecCare Dent. 2000;20(1):23-27.

31. Arvidson-Bufano UB, Blank LW, Yellowitz JA. Nurses' oral health assessments of nursing home residents pre- and post-training: a pilot study. Spec Care Dentist. 1996;16(2):58-64.

32. Guay AH. The oral health status of nursing home residents: what do we need to know? $J$ Dent Educ. 2005;69(9):1015-1017.

33. Hawes C, Morris JN, Phillips CD, Mor V, Fries BE, Nonemaker S. Reliability Estimates for The Minimum Data Set for Nursing Home Resident Assessment and Care Screening ( MDS ) 1. Gerontologist. 1995;35(2):172-178.

34. Flemisch Government. Flemish government resolutions 2016.

http://www.ejustice.just.fgov.be/cgi_loi/change_lg.pl?language=nl\&la=N\&table_name=wet\&c $\mathrm{n}=2016051323$. Accessed December 14, 2016.

35. Desomer A, Mistiaen P, Eyssen M. Belrai Suite of Instruments: An Exploratory Study on Applicability for Individual Care Planning and Budget Allocation in Rehabilitation Care 2016.; 2016.

36. Vanneste D, De Almeida Mello J, Macq J, Van Audenhove C DA. Incomplete assessments: towards a better understanding of causes and solutions. The case of the interRAI home care instrument in Belgium. PLoS One. 2015. http://dx.plos.org/10.1371/journal.pone.0123760.

37. De Almeida Mello J, Van Durme T, Macq J, Declercq A. Interventions to delay institutionalization of frail older persons: design of a longitudinal study in the home care setting. BMC Public Health. 2012;12(1):615. doi:10.1186/1471-2458-12-615

38. Lynn Mary R. Determination and Quantification of Content Validity. Nurs Res. 1986;35(6):382-386.

39. Polit DF, Beck CT. The content validity index: are you sure you know what's being reported? Critique and recommendations. Res Nurs Health. 2006;29(5):489-497. doi:10.1002/nur.20147

40. Polit DF, Beck CT, Owen S V. Is the CVI an acceptable indicator of content validity? Appraisal and recommendations. Res Nurs Health. 2007;30(4):459-467. doi:10.1002/nur.20199

41. Krueger RA, Casey MA. Focus Groups: A Practical Guide for Applied Research (3rd Ed.). 3rd ed. Thousand Oaks, CA: Sage; 2000.

42. Leung F, Savithiri R. Spotlight on focus groups. Can Fam Physician. 2009;55:218-219.

43. Mayring Philipp. Qualitative Content Analysis.; 2014. doi:10.1016/S1479-3709(07)11003-7

44. Juthani-Mehta $\mathrm{M}$, De Rekeneire $\mathrm{N}$, Allore $\mathrm{H}$, et al. Modifiable risk factors for pneumonia requiring hospitalization of community-dwelling older adults: the Health, Aging, and Body Composition Study. J Am Geriatr Soc. 2013;61(7):1111-1118. doi:10.1111/jgs.12325

45. Bassim CW, Gibson G, Ward T, Paphides BM, Denucci DJ. Modification of the risk of mortality from pneumonia with oral hygiene care. J Am Geriatr Soc. 2008;56(9):1601-1607. 
doi:10.1111/j.1532-5415.2008.01825.x

46. Azarpazhooh A, Leake JL. Systematic review of the association between respiratory diseases and oral health. J Periodontol. 2006;77(September):1465-1482. doi:10.1902/jop.2006.060010

47. Lexomboon D, Trulsson M, Wårdh I, Parker MG. Chewing ability and tooth loss: association with cognitive impairment in an elderly population study. J Am Geriatr Soc. 2012;60(10):1951-1956. doi:10.1111/j.1532-5415.2012.04154.x

48. Takata $\mathrm{Y}$, Ansai $\mathrm{T}$, Awano $\mathrm{S}$, et al. Relationship of physical fitness to chewing in an 80-yearold population. Oral Dis. 2004;10(1):44-49. http://www.ncbi.nlm.nih.gov/pubmed/14996294.

49. Kimura $\mathrm{Y}$, Ogawa $\mathrm{H}$, Yoshihara $\mathrm{A}$, et al. Evaluation of chewing ability and its relationship with activities of daily living, depression, cognitive status and food intake in the communitydwelling elderly. Geriatr Gerontol Int. 2013;13(3):718-725. doi:10.1111/ggi.12006

50. Southerland JH, Moss K, Taylor GW, et al. Periodontitis and diabetes associations with measures of atherosclerosis and CHD. Atherosclerosis. 2012;222(1):196-201. doi:10.1016/j.atherosclerosis.2012.01.026

51. $\mathrm{Yu}$ Y-H, Kuo H-K. Association between cognitive function and periodontal disease in older adults. J Am Geriatr Soc. 2008;56(9):1693-1697. doi:10.1111/j.1532-5415.2008.01847.x

52. Avlund K, Holm-Pedersen P, Morse DE, Viitanen M, Winblad B. Tooth loss and caries prevalence in very old Swedish people: the relationship to cognitive function and functional ability. Gerodontology. 2004;21(1):17-26. http://www.ncbi.nlm.nih.gov/pubmed/15074536.

53. Chen X, Clark JJ, Chen H, Naorungroj S. Cognitive impairment, oral self-care function and dental caries severity in community-dwelling older adults. Gerodontology. June 2013:1-9. doi:10.1111/ger.12061

54. Syrjälä A-MH, Ylöstalo $P$, Sulkava R, Knuuttila M. Relationship between cognitive impairment and oral health: results of the Health 2000 Health Examination Survey in Finland. Acta Odontol Scand. 2007;65(2):103-108. doi:10.1080/00016350601083521

55. Moriya S, Notani K, Miura H, Inoue N. Relationship between masticatory ability and physical performance in community-dwelling edentulous older adults wearing complete dentures. Gerodontology. December 2012:1-9. doi:10.1111/ger.12029

56. Lee I-C, Yang Y-H, Ho P-S. Chewing ability, nutritional status and quality of life. J Oral Rehabil. 2014;41(2):79-86. doi:10.1111/joor.12115

57. Stenman U, Ahlqwist M, Björkelund C, Hakeberg M. Oral health-related quality of life-associations with oral health and conditions in Swedish 70-year-old individuals. Gerodontology. 2012;29(2):e440-6. doi:10.1111/j.1741-2358.2011.00495.x

58. Takata Y, Ansai T, Awano S, et al. Chewing ability and quality of life in an 80-year-old population. J Oral Rehabil. 2006;33(5):330-334. doi:10.1111/j.1365-2842.2005.01567.x

59. Kimura $\mathrm{Y}$, Wada $\mathrm{T}$, Ishine $\mathrm{M}$, et al. Community-dwelling elderly with chewing difficulties are more disabled, depressed and have lower quality of life scores. Geriatr Gerontol Int. 2009;9(1):102-104. doi:10.1111/j.1447-0594.2008.00489.x 
60. Chen H, linuma M, Onozuka M, Kubo K-Y. Chewing Maintains Hippocampus-Dependent Cognitive Function. Int J Med Sci. 2015;12(6):502-509. doi:10.7150/ijms.11911

61. Pistorius J, Horn JG, Pistorius A, Kraft J. Oral health-related quality of life in patients with removable dentures. Schweiz Monatsschr Zahnmed. 2013;123(11):964-71; 955.

62. Ha JE, Heo YJ, Jin BH, Paik DI, Bae KH. The impact of the National Denture Service on oral health-related quality of life among poor elders. J Oral Rehabil. 2012;(6). doi:10.1111/j.13652842.2012.02296.x

63. Michaud P-L, de Grandmont P, Feine JS, Emami E. Measuring patient-based outcomes: is treatment satisfaction associated with oral health-related quality of life? $J$ Dent. 2012;40(8):624-631. doi:10.1016/j.jdent.2012.04.007

64. Ikebe K, Matsuda KI, Morii K, et al. Impact of dry mouth and hyposalivation on oral healthrelated quality of life of elderly Japanese. Oral Surgery, Oral Med Oral Pathol Oral Radiol Endodontology. 2007;103(2):216-222. doi:10.1016/j.tripleo.2005.12.001

65. Gerdin EW, Einarson S, Jonsson M, Aronsson K, Johansson I. Impact of dry mouth conditions on oral health-related quality of life in older people. Gerodontology. 2005;22(4):219-226.

66. Hahnel S, Schwarz S, Zeman F, Schäfer L, Behr M. Prevalence of xerostomia and hyposalivation and their association with quality of life in elderly patients in dependence on dental status and prosthetic rehabilitation: A pilot study. J Dent. 2014;42(6):664-670. doi:10.1016/j.jdent.2014.03.003

67. Dellefield ME. Implementation of the resident assessment instrument/minimum data set in the nursing home as organization: implications for quality improvement in RN clinical assessment. Geriatr Nurs. 2007;28(6):377-386. doi:10.1016/j.gerinurse.2007.03.002

68. Rahman AN, Applebaum RA. The nursing home Minimum Data Set assessment instrument: manifest functions and unintended consequences--past, present, and future. Gerontologist. 2009;49(6):727-735. doi:10.1093/geront/gnp066

69. Villa A, Connell CL, Abati S. Diagnosis and management of xerostomia and hyposalivation. Ther Clin Risk Manag. 2015;11:45-51. doi:10.2147/TCRM.S76282

70. Chalmers JM, King PL, Spencer a J, Wright F a C, Carter KD. The oral health assessment tool--validity and reliability. Aust Dent J. 2005;50(3):191-199.

71. De Visschere L, de Baat C, De Meyer L, et al. The integration of oral health care into day-today care in nursing homes: a qualitative study. Gerodontology. 2013:10-13.

doi:10.1111/ger.12062

72. Walker KK, Jackson RD. Oral health beliefs and behaviors of nurse and nurse practitioner students using the HU-DBI inventory: An opportunity for oral health vicarious learning. $J$ Nurs Educ Pract. 2017;7(8):19. doi:10.5430/jnep.v7n8p19

73. Hilton S, Class B, Sheppard JJ, Hemsley B. Feasibility of implementing oral health guidelines in residential care settings: views of nursing staff and residential care workers. Appl Nurs Res. 2016;30:194-203. doi:10.1016/j.apnr.2015.10.005 
74. Chung. Dental care of elderly in nursing homes: perceptions of managers, nurses, and physicians. 2000;20(1):12-17.

75. Grant AM. The significance of task significance: Job performance effects, relational mechanisms, and boundary conditions. J Appl Psychol. 2008;93(1):108-124. doi:10.1037/0021-9010.93.1.108

76. Bandura A, Locke EA. Negative self-efficacy and goal effects revisited. J Appl Psychol. 2003;88(1):87-99. doi:10.1037/0021-9010.88.1.87

77. Paulsson G, Nederfors T, Fridlund B. Nursing personnel's views on oral health from a health promotion perspective: a grounded theory analysis. 2002;6357(December). doi:10.1080/000163502753471998 